\title{
Diversity of soil nematodes in meadows of the White Carpathians
}

\author{
L. HÁNĚL ${ }^{1}$, A. ČEREVKOVÁ ${ }^{2}$
}

\begin{abstract}
${ }^{1}$ Biological Centre, Academy of Sciences of the Czech Republic, Institute of Soil Biology, Na Sádkách 7, CZ-370 05 České Budějovice, Czech Republic, E-mail: hanel@upb.cas.cz; ${ }^{2}$ Parasitological Institute of the Slovak Academy of Sciences, Hlinkova 3, 04001 Košice, Slovak Republic, E-mail: cerev@saske.sk
\end{abstract}

\begin{abstract}
Summary
Soil nematodes were studied in 11 meadow sites in the White Carpathians. In total, 155 species and 86 genera were found but the composition of nematode assemblages in eastern and western side of the mountains differed. Most nematode species belonged to the orders Tylenchida (52), Dorylaimida (35) and Rhabditida (16), and to the trophic groups bacterivores (47), plant parasites (29) and omnivores (25). In individual sites and sampling dates the number of species varied in range $18-68$, genera $18-52, \mathrm{H}$ 'spp 1.32 - 3.50, H'gen 1.31 - 3.21, T $1.79-5.70$, MI $2.32-$ 3.63, PPI 2.03 - 2.98, and $\Sigma$ MI 2.20 - 3.50. Composition of nematode assemblages and their matrix classification indicated a low degree of anthropogenous disturbance to the meadows.
\end{abstract}

Key words: Nematoda; diversity; trophic groups; grassland; meadow

\section{Introduction}

Nematodes are important group of soil animals that influence processes in European grasslands (Ekschmitt et al., 2001). In Central Europe, greater part of meadows was established by human activity. Diversity of soil nematodes in meadows may be greater than in (sub) climax forests (Háněl, 1995), nevertheless, can be affected by meadow management (Yeates \& Bongers, 1999). The nematode fauna in the White Carpathians is practically unknown, therefore meadows on this territory were included in the project aimed at study of grassland nematodes in the Czech Republic and the Slovak Republic. The goal of this paper is to study diversity soil nematodes in selected meadow habitats and evaluate community indices that are used to characterize nematode communities.

\section{Material and Methods}

Nematodes were studied in seven localities of the White Carpathians.
Locality Vrbovce \& Vrbovce-Šance, $360 \mathrm{~m}$ a.s.1., $48^{\circ} 47^{\prime} \mathrm{N}$, $17^{\circ} 28^{\prime} \mathrm{E}$, gravely loam soil, samples taken in three sites:

1: old meadow, moderately mown; $\mathrm{pH}\left(\mathrm{H}_{2} \mathrm{O}\right) 7.00, \mathrm{C}_{\mathrm{ox}} 5.22$ \%; plant cover: Acetosa pratensis Mill., Trifolium L., Achillea L., Cirsium arvense (L.), Festuca rubra L., Agrostis L. and others; sampling date: October 2004.

2: meadow on previously arable soil, mown; $\mathrm{pH}\left(\mathrm{H}_{2} \mathrm{O}\right)$ $5.30, \mathrm{C}_{\mathrm{ox}} 4.37 \%$; plant cover: Plantago lanceolata L., Dactylis glomerata L., F. rubra L., Trifolium L., Agrostis L. and others; sampling date: October 2004.

3: pasture meadow; $\mathrm{pH}\left(\mathrm{H}_{2} \mathrm{O}\right) 7.06, \mathrm{C}_{\mathrm{ox}} 6.16 \%$; plant cover: Senecio L., Brachypodium P. Beauv., P. lanceolata L., D. glomerata L., Trifolium repens L., T. pratense L., Taraxacum officinale Web. in Wiggers and others; sampling date: October 2004.

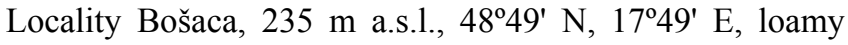
sand soil, samples taken in two sites:

4: original meadow, recently mown; $\mathrm{pH}\left(\mathrm{H}_{2} \mathrm{O}\right) 8.86, \mathrm{C}_{\mathrm{ox}}$ $5.91 \%$; plant cover: Agrimonia eupatoria L., Festuca L., Arrhenatherum elatius (L.) P. Beauv., Fragaria vesca L. and others.

5: pasture meadow; $\mathrm{pH}\left(\mathrm{H}_{2} \mathrm{O}\right) 7.82, \mathrm{C}_{\mathrm{ox}} 6.58 \%$; plant cover: Ononis spinosa L., Festuca L., Dactylis L., Agros-tis L. and others; sampling date: October 2004.

Locality Hrabovka, 260 m a.s.1., 490 $07^{\prime} \mathrm{N}, 18^{\circ} 18^{\prime} \mathrm{E}$, loam soil, samples taken in one site:

6: pasture meadow; $\mathrm{pH}\left(\mathrm{H}_{2} \mathrm{O}\right) 7.15, \mathrm{C}_{\mathrm{ox}} 3.15 \%$; plant cover: T. officinale Web. in Wiggers, Cichorium intybus L., Acetosa pratensis Mill, Vicia L., E. repens (L.) Desv._and others; sampling date: October 2004.

Locality Vršatecké podhradie, $553 \mathrm{~m}$ a.s.1., 490' $\mathrm{N}, 18^{\circ} 08^{\prime}$ $\mathrm{E}$, loam soil and clay loam soil, samples taken in two sites: 7: pasture meadow; loam soil, $\mathrm{pH}\left(\mathrm{H}_{2} \mathrm{O}\right) 8.96, \mathrm{C}_{\mathrm{ox}} 4.44 \%$; plant cover: E. repens (L.) Desv., Potentilla L., Myurella tenerrima (Brid.) Lindb., C. arvense (L.) Scop., P. lanceolata L., Senecio L. and others; sampling date: October 
2004.

8: original meadow; clay loam soil, $\mathrm{pH}\left(\mathrm{H}_{2} \mathrm{O}\right) 7.82, \mathrm{C}_{\text {ox }} 5.05$ \%; plant cover: A. elatius (L.) P. Beauv. ex J. Presl et C. Presl, Brachypodium P. Beauv., E. repens (L.), Potentil-la (L.), C. arvense (L.) Scop. and others; sampling date: October 2004.

Locality Červený kameň, $335 \mathrm{~m}$ a.s.1., $49^{\circ} 04^{\prime} \mathrm{N}, 18^{\circ} 10^{\prime} \mathrm{E}$, clay loam soil, samples taken in one site:

9: intensively managed meadow, mown: $\mathrm{pH}\left(\mathrm{H}_{2} \mathrm{O}\right) 7.66$, $\mathrm{C}_{\text {ox }} 5.78 \%$; plant cover: F. rubra L., A. stolonifera L., Lolium perenne L., E. repens (L.) Desv. and others; sampling date: October 2004.

Locality Mikušovce, $350 \mathrm{~m}$ a.s.1., $49^{\circ} 04^{\prime} \mathrm{N}, 18^{\circ} 13^{\prime} \mathrm{E}$, loamy sand soil, samples taken in one site:

10: meadow with solitary trees; $\mathrm{pH}\left(\mathrm{H}_{2} \mathrm{O}\right) 8.88, \mathrm{C}_{\mathrm{ox}} 4.27 \%$; plant cover: F. rubra L., A. elatius (L.) P. Beauv. ex J. Presl et C. Presl, and others with solitary of Malus Mill.; sampling date: October 2004.

Locality Čertoryje (samples C1 - C7), National Nature Reserve, $370 \mathrm{~m}$ a.s.l., $48^{\circ} 54^{\prime} \mathrm{N}, 17^{\circ} 25^{\prime} \mathrm{E}$, clay-loam soil, $\mathrm{pH}$ $\left(\mathrm{H}_{2} \mathrm{O}\right) 5.30, \mathrm{C}_{\mathrm{ox}} 4.37 \%$; plant cover: a species-rich subthermophilous meadow of the association BrachypodioMolinietum Klika 1939, dominant plants mainly grasses such as Bromus erectus Huds., Brachypodium pinnatum (L.) P. Beauv., Molinia arundinacea Schrank, Festuca rupicola Heuffel, sedge Carex montana L. accompanied by herbs Potentilla alba L., Cirsium pannonicum (L.) Link, Viola hirta L., Astragalus danicus Retz., Polygala major Jacq., Filipendula vulgaris Moench, Geranium sengui-neum
L., Centaurea scabiosa L., Serratula tinctoria L., Salvia pratensis L., and others with solitary and small groups of Quercus robur $\mathrm{L}$. trees and various shrubs; sam-pling dates: 16 November 1999 (C1), 23 May 2000 (C2), 5 September 2000 (C3), 14 November 2000 (C4), 9 May 2001 (C5), 4 September 2001 (C6), and 12 November 2001 (C7). Details see in Háněl (2003).

Nematodes were isolated from four 10 -g sub-samples of the mixed wet soil using modified Baermann funnels, killed and fixed in $3 \%$ formaldehyde and studied in glycerine slides. Data in Table 1 express nematode individuals in $40 \mathrm{~g}$ of soil.

Nematode species were allocated to trophic and c-p groups according to Yeates et al. (1993) and Bongers (1990). Community indices were calculated as suggested by Freckman and Ettema (1993) and Yeates and Bongers (1999): Shannon Index (H'spp, H'gen and H'tg calculated for species, genera and trophic groups, respectively; with natural logarithm), richness Margalef Index (SR for species, GR for genera), Simpson Index (Dspp for species, Dgen for genera), Trophic Diversity Heip's et al. (1988) Index (T for trophic groups), Maturity Index (MI), Plant Parasite In-dex (PPI; all species of the order Tylenchida and the plantparasitic Dorylaimida included), and $\Sigma$ Maturity Index ( $\mathrm{MI}$ ). Channel Index (CI), Enrichment Index (EI) and Structure Index (SI) were calculated according to Ferris et al. (2001) with weightings of nematode taxa as suggested by the authors. The weightings for the taxa not included in the paper were derived from c-p values by Bongers (1990). Statistical calculations were performed using the STATISTICA (StatSoft, 2001).

Table 1. Population densities (individuals in $40 \mathrm{~g}$ of fresh soil) of soil nematode species in the White Carpathians: mean density (mean), standard deviation ( $\mathrm{SD}, \mathrm{n}=17)$, dominance $(\mathrm{D}[\%])$, frequency of occurrence $(\mathrm{F}[\%])$; $\mathrm{TG}$ - trophic groups bacterivores $(\mathrm{B})$, fungivores $(\mathrm{F})$, root-fungal feeders (RFF), plant parasites $(\mathrm{PP})$, omnivores $(\mathrm{O})$, predators $(\mathrm{P})$, insect parasites (IP)

\begin{tabular}{|c|c|c|c|c|c|c|c|}
\hline No. & TG & c-p & Species in orders & mean & $\mathrm{SD}$ & $\mathrm{D}[\%]$ & $\mathrm{F}[\%]$ \\
\hline & & & MONHYSTERIDA & & & & \\
\hline & & & Eumonhystera longicaudatula (Gerlach & & & & \\
\hline 1 & B & 1 & et Riemann, 1973) & 0.1 & 0.5 & 0.04 & 5.88 \\
\hline 2,3 & B & 1 & Geomonhystera australis (Cobb, 1893) \& villosa (Bütschli, 1873) & 0.5 & 1.2 & 0.18 & 23.53 \\
\hline 4 & B & 1 & Monhystrella cf paramacrura (Meyl, 1953) & 0.4 & 1.2 & 0.13 & 11.76 \\
\hline 5 & B & 1 & $\begin{array}{l}\text { Theristus sp. } \\
\text { CHROMADORIDA }\end{array}$ & 0.2 & 0.5 & 0.07 & 11.76 \\
\hline 6 & B & 3 & Achromadora ruricola (de Man, 1880) & 0.1 & 0.2 & 0.02 & 5.88 \\
\hline 7 & B & 3 & $\begin{array}{l}\text { Prodesmodora arctica (Mulvey, 1969) } \\
\text { ARAEOLAIMIDA }\end{array}$ & 0.1 & 0.3 & 0.04 & 11.76 \\
\hline 8 & B & 2 & Cylindrolaimus communis de Man, 1880 & 0.2 & 0.4 & 0.07 & 17.65 \\
\hline 9 & B & 2 & Anaplectus granulosus (Bastian, 1865) & 3.5 & 4.4 & 1.33 & 52.94 \\
\hline 10 & B & 2 & Plectus acuminatus Bastian, 1865 & 1.2 & 1.9 & 0.47 & 41.18 \\
\hline 11 & B & 2 & Plectus communis Bütschli, 1873 & 0.4 & 0.8 & 0.16 & 23.53 \\
\hline 12 & B & 2 & Plectus geophilus de Man, 1880 & 0.1 & 0.3 & 0.04 & 11.76 \\
\hline 13 & B & 2 & Plectus opisthocirculus Andrássy, 1952 & 0.5 & 2.2 & 0.20 & 5.88 \\
\hline 14 & B & 2 & Plectus parietinus Bastian, 1865 & 2.2 & 2.4 & 0.84 & 88.24 \\
\hline 15 & B & 2 & Plectus parvus Bastian, 1865 & 0.4 & 1.1 & 0.16 & 17.65 \\
\hline 16 & B & 2 & Plectus rhizophilus de Man, 1880 & 0.5 & 1.3 & 0.20 & 23.53 \\
\hline
\end{tabular}




\begin{tabular}{|c|c|c|c|c|c|c|c|}
\hline 17 & $\mathrm{~B}$ & 2 & Plectus thornei Rühm, 1956 & 0.5 & 1.9 & 0.20 & 11.76 \\
\hline 18 & $\mathrm{~B}$ & 2 & Ceratoplectus armatus (Bütschli, 1873) & 0.4 & 0.7 & 0.13 & 23.53 \\
\hline 19 & $\mathrm{~B}$ & 2 & Tylocephalus auriculatus (Bütschli, 1873) & 0.2 & 0.7 & 0.07 & 5.88 \\
\hline 20 & B & 2 & Tylocephalus laticollis Zell, 1985 & 0.1 & 0.2 & 0.02 & 5.88 \\
\hline 21 & $\mathrm{~B}$ & 2 & $\begin{array}{l}\text { Wilsonema otophorum (de Man, 1880) } \\
\text { TERATOCEPHALIDA }\end{array}$ & 0.1 & 0.2 & 0.02 & 5.88 \\
\hline 22 & $\mathrm{~B}$ & 3 & $\begin{array}{l}\text { Metateratocephalus crassidens (de Man, 1880) } \\
\text { RHABDITIDA }\end{array}$ & 0.1 & 0.3 & 0.04 & 11.76 \\
\hline 23 & $\mathrm{~B}$ & 2 & Heterocephalobus elongatus (de Man, 1880) & 4.0 & 13.2 & 1.51 & 29.41 \\
\hline 24 & B & 2 & $\begin{array}{l}\text { Cephalobus persegnis Bastian, } 1865 \\
\text { Eucephalobus mucronatus (Kozlowska }\end{array}$ & 10.1 & 12.9 & 3.82 & 82.35 \\
\hline 25 & B & 2 & et Roguska-Wasilewska, 1963) & 0.9 & 1.2 & 0.33 & 41.18 \\
\hline 26 & $\mathrm{~B}$ & 2 & Eucephalobus oxyuroides (de Man, 1876) & 2.9 & 5.5 & 1.09 & 52.94 \\
\hline 27 & B & 2 & Eucephalobus striatus (Bastian, 1865) & 3.5 & 6.3 & 1.33 & 64.71 \\
\hline 28 & B & 2 & Acrobeloides nanus (de Man, 1880) & 9.6 & 9.3 & 3.62 & 76.47 \\
\hline 29 & $\mathrm{~B}$ & 2 & Chiloplacus propinquus (de Man, 1921) & 0.4 & 1.2 & 0.16 & 17.65 \\
\hline 30 & B & 2 & Chiloplacus symmetricus (Thorne, 1925) & 0.4 & 1.2 & 0.16 & 11.76 \\
\hline 31 & B & 2 & Cervidellus neftasiensis Boström, 1986 & 0.1 & 0.2 & 0.02 & 5.88 \\
\hline 32 & $\mathrm{~B}$ & 1 & Panagrolaimus rigidus (Schneider, 1866) & 6.1 & 15.7 & 2.31 & 41.18 \\
\hline 33 & $\mathrm{~B}$ & 1 & Rhabditis cf. terricola Dujardin, 1845 & 12.9 & 14.9 & 4.86 & 88.24 \\
\hline 34 & B & 1 & Bursilla monhystera (Bütschli, 1873) & 1.1 & 2.9 & 0.40 & 23.53 \\
\hline 35 & B & 1 & Diploscapter cf coronatus (Cobb, 1893) & 0.4 & 1.2 & 0.13 & 11.76 \\
\hline 36 & IN & 1 & Heterorhabditis sp. juvs. & 0.2 & 0.7 & 0.07 & 5.88 \\
\hline 37 & IN & 1 & Steinernema affine (Bovien, 1937) & 0.2 & 0.7 & 0.07 & 5.88 \\
\hline 38 & IN & 1 & $\begin{array}{l}\text { Steinernema juvs. } \\
\text { APHELENCHIDA }\end{array}$ & 0.2 & 0.6 & 0.09 & 17.65 \\
\hline 39 & $\mathrm{~F}$ & 2 & Aphelenchus avenae Bastian, 1865 & 6.8 & 7.6 & 2.55 & 88.24 \\
\hline 40 & $\mathrm{~F}$ & 2 & Paraphelenchus pseudoparietinus Micoletzky, 1922 & 1.2 & 2.5 & 0.44 & 23.53 \\
\hline 41 & $\mathrm{~F}$ & 2 & Aphelenchoides bicaudatus (Imamura, 1931) & 0.7 & 2.0 & 0.27 & 23.53 \\
\hline 42 & $\mathrm{~F}$ & 2 & Aphelenchoides composticola Franklin, 1957 & 0.7 & 1.1 & 0.27 & 35.29 \\
\hline 43 & $\mathrm{~F}$ & 2 & Aphelenchoides curiolis Gritsenko, 1971 & 6.5 & 11.4 & 2.44 & 41.18 \\
\hline 44 & $\mathrm{~F}$ & 2 & Aphelenchoides saprophilus Franklin, 1957 & 1.4 & 2.7 & 0.53 & 29.41 \\
\hline 45 & $\mathrm{~F}$ & 2 & Aphelenchoides spicomucronatus Truskova, 1973 & 0.8 & 1.5 & 0.31 & 35.29 \\
\hline 46 & $\mathrm{~F}$ & 2 & $\begin{array}{l}\text { Aphelenchoides } s p \text {. } \\
\text { TYLENCHIDA }\end{array}$ & 0.3 & 0.8 & 0.11 & 11.76 \\
\hline 47 & RFF & 2 & Psilenchus hilarulus de Man, 1921 & 0.2 & 0.5 & 0.07 & 11.76 \\
\hline 48 & RFF & 2 & Basiria gracilis (Thorne, 1949) & 0.5 & 0.9 & 0.18 & 23.53 \\
\hline 49 & RFF & 2 & Basiria graminophila Siddiqi, 1959 & 0.1 & 0.5 & 0.04 & 5.88 \\
\hline 50 & RFF & 2 & Boleodorus thylactus (Thorne, 1941) & 3.2 & 6.3 & 1.22 & 35.29 \\
\hline 51 & RFF & 2 & Neopsilenchus magnidens (Thorne, 1949) & 0.8 & 1.4 & 0.31 & 35.29 \\
\hline 52 & RFF & 2 & Filenchus discrepans (Andrássy, 1954) & 0.2 & 0.6 & 0.09 & 17.65 \\
\hline 53 & RFF & 2 & Filenchus filiformis (Bütschli, 1873) - vulgaris (Brzeski, 1963) & 5.5 & 5.3 & 2.09 & 88.24 \\
\hline 54 & RFF & 2 & Filenchus hamatus (Thorne et Malek, 1968) & 0.1 & 0.5 & 0.04 & 5.88 \\
\hline 55 & RFF & 2 & Filenchus longicaudatulus Zell, 1988 & 0.2 & 0.5 & 0.07 & 11.76 \\
\hline 56 & RFF & 2 & Filenchus misellus (Andrássy, 1958) & 35.6 & 59.2 & 13.45 & 41.18 \\
\hline 57 & RFF & 2 & Filenchus polyhypnus (Steiner et Albin, 1946) & 0.2 & 0.7 & 0.07 & 5.88 \\
\hline 58 & RFF & 2 & Filenchus sp. & 0.1 & 0.3 & 0.04 & 11.76 \\
\hline 59 & RFF & 2 & Tylenchus davainei Bastian, 1865 & 0.1 & 0.2 & 0.02 & 5.88 \\
\hline 60 & RFF & 2 & Tylenchus elegans de Man, 1876 & 0.1 & 0.2 & 0.02 & 5.88 \\
\hline 61 & RFF & 2 & Tylenchus sp. juvs. & 0.4 & 0.9 & 0.13 & 17.65 \\
\hline 62 & RFF & 2 & Miculenchus salvus Andrássy, 1959 & 0.1 & 0.2 & 0.02 & 5.88 \\
\hline 63 & RFF & 2 & Malenchus andrassyi Merny, 1970 & 0.1 & 0.2 & 0.02 & 5.88 \\
\hline 64 & RFF & 2 & Malenchus cognatus Andrássy, 1981 & 0.1 & 0.5 & 0.04 & 5.88 \\
\hline 65 & RFF & 2 & Malenchus exiguus (Massey, 1969) & 0.6 & 2.0 & 0.24 & 17.65 \\
\hline 66 & RFF & 2 & Coslenchus alacinatus Siddiqi, 1981 & 0.6 & 1.7 & 0.24 & 23.53 \\
\hline 67 & RFF & 2 & Coslenchus costatus (de Man, 1921) & 0.6 & 1.2 & 0.22 & 23.53 \\
\hline 68 & RFF & 2 & Aglenchus agricola (de Man, 1884) & 4.6 & 8.4 & 1.75 & 52.94 \\
\hline
\end{tabular}




\begin{tabular}{|c|c|c|c|c|c|c|c|}
\hline 69 & $\mathrm{~F}$ & 2 & Ditylenchus acutus (Khan, 1965) & 0.3 & 0.8 & 0.11 & 11.76 \\
\hline 70 & $\mathrm{~F}$ & 2 & Ditylenchus medicaginis Wasilewska, 1965 & 2.8 & 3.8 & 1.04 & 41.18 \\
\hline 71 & $\mathrm{~F}$ & 2 & Nothotylenchus acris Thorne, 1941 & 0.1 & 0.5 & 0.04 & 5.88 \\
\hline 72 & PP & 2 & Bitylenchus dubius (Bütschli, 1873) & 2.1 & 3.7 & 0.78 & 41.18 \\
\hline 73 & PP & 3 & Tylenchorhynchus cylindricus Cobb, 1913 & 0.8 & 2.2 & 0.31 & 23.53 \\
\hline 74 & PP & 3 & Tylenchorhynchus sp. juvs. & 0.2 & 0.5 & 0.07 & 11.76 \\
\hline 75 & PP & 3 & Amplimerlinius globigerus Siddiqi, 1979 & 0.9 & 3.4 & 0.33 & 11.76 \\
\hline 76 & PP & 3 & Amplimerlinius macrurus (Goodey, 1932) & 5.0 & 20.1 & 1.89 & 11.76 \\
\hline 77 & PP & 3 & Trophurus sculptus Loof, 1956 & 0.8 & 2.1 & 0.31 & 17.65 \\
\hline 78 & PP & 3 & Rotylenchus goodeyi Loof et Oostenbrink, 1958 & 0.5 & 1.2 & 0.18 & 17.65 \\
\hline 79 & PP & 3 & Rotylenchus robustus (de Man, 1876) & 0.3 & 1.0 & 0.11 & 11.76 \\
\hline 80 & PP & 3 & $\begin{array}{l}\text { Helicotylenchus canadensis Waseem, } 1961 \\
\text { Helicotylenchus digonicus Perry in Perry, Darling et Thorne, }\end{array}$ & 4.9 & 9.3 & 1.86 & 47.06 \\
\hline 81 & PP & 3 & 1959 & 26.2 & 61.1 & 9.87 & 58.82 \\
\hline 82 & PP & 3 & Helicotylenchus pseudorobustus (Steiner, 1914) & 0.2 & 0.7 & 0.09 & 11.76 \\
\hline 83 & PP & 3 & Helicotylenchus vulgaris Yuen, 1964 & 0.2 & 0.5 & 0.07 & 11.76 \\
\hline 84 & PP & 3 & Pratylenchus crenatus Loof, 1960 & 0.5 & 0.9 & 0.20 & 29.41 \\
\hline 85 & PP & 3 & Pratylenchus pratensis (de Man, 1880) & 1.1 & 2.3 & 0.42 & 29.41 \\
\hline 86 & PP & 2 & Paratylenchus bukowinensis Micoletzky, 1922 & 2.1 & 5.2 & 0.78 & 23.53 \\
\hline 87 & PP & 2 & Paratylenchus goodeyi Oostenbrink, 1953 & 0.1 & 0.5 & 0.04 & 5.88 \\
\hline 88 & PP & 2 & Paratylenchus microdorus Andrássy, 1959 & 0.2 & 1.0 & 0.09 & 5.88 \\
\hline 89 & PP & 2 & Paratylenchus similis Khan, Prasad et Mathur, 1967 & 0.1 & 0.2 & 0.02 & 5.88 \\
\hline 90 & PP & 2 & Paratylenchus steineri Golden, 1961 & 0.9 & 2.1 & 0.33 & 23.53 \\
\hline 91 & PP & 2 & Paratylenchus straeleni (de Coninck, 1931) & 0.4 & 1.7 & 0.16 & 5.88 \\
\hline 92 & PP & 2 & Paratylenchus sp.1 & 0.1 & 0.5 & 0.04 & 5.88 \\
\hline 93 & PP & 2 & Paratylenchus sp.2 juvs. & 0.4 & 1.3 & 0.16 & 11.76 \\
\hline 94 & PP & 3 & Criconemoides parvus Raski, 1952 & 0.4 & 1.7 & 0.16 & 5.88 \\
\hline 95 & PP & 3 & Sphaeronema juvs. & 0.9 & 2.3 & 0.36 & 17.65 \\
\hline 96 & PP & 3 & Xenocriconemella macrodora (Taylor, 1936) & 0.4 & 1.0 & 0.16 & 17.65 \\
\hline 97 & PP & 3 & Mesocriconema antipolitanum (de Guiran, 1963) & 0.2 & 0.4 & 0.07 & 17.65 \\
\hline 98 & PP & 3 & $\begin{array}{l}\text { Mesocriconema rusticum (Micoletzky, 1915) } \\
\text { ENOPLIDA }\end{array}$ & 0.1 & 0.5 & 0.04 & 5.88 \\
\hline 99 & $\mathrm{~B}$ & 3 & Bastiania gracilis de Man, 1876 & 0.8 & 2.0 & 0.31 & 29.41 \\
\hline 100 & $\mathrm{~B}$ & 3 & Odontolaimus chlorurus de Man, 1880 & 0.4 & 0.9 & 0.13 & 17.65 \\
\hline 101 & $\mathrm{P}$ & 3 & Tripyla filicaudata de Man, 1880 & 2.0 & 5.6 & 0.75 & 23.53 \\
\hline 102 & $\mathrm{P}$ & 3 & Tripyla setifera Bütschli, 1873 & 0.4 & 1.0 & 0.16 & 23.53 \\
\hline 103 & $\mathrm{~B}$ & 3 & Prismatolaimus dolichurus de Man, 1880 & 0.1 & 0.2 & 0.02 & 5.88 \\
\hline 104 & $\mathrm{~B}$ & 3 & Prismatolaimus intermedius (Bütschli, 1873) & 1.8 & 3.9 & 0.69 & 41.18 \\
\hline 105 & $\mathrm{~B}$ & 3 & Prismatolaimus matoni Mulk et Coomans, 1979 & 0.4 & 1.2 & 0.13 & 11.76 \\
\hline 106 & B & 3 & $\begin{array}{l}\text { Aulolaimus nannocephalus Andrássy, } 1972 \\
\text { ALAIMIDA }\end{array}$ & 0.1 & 0.2 & 0.02 & 5.88 \\
\hline 107 & $\mathrm{~B}$ & 4 & Alaimus arcuatus Thorne, 1939 & 0.1 & 0.2 & 0.02 & 5.88 \\
\hline 108 & $\mathrm{~B}$ & 4 & Alaimus meyli Andrássy, 1961 & 0.1 & 0.2 & 0.02 & 5.88 \\
\hline 109 & $\mathrm{~B}$ & 4 & Alaimus primitivus de Man, 1880 & 1.4 & 3.6 & 0.53 & 35.29 \\
\hline 110 & $\mathrm{~B}$ & 4 & Paramphidelus dolichurus (de Man, 1876) & 0.2 & 0.7 & 0.07 & 5.88 \\
\hline 111 & $\mathrm{~B}$ & 4 & Paramphidelus pseudobulbosus (Altherr, 1953) & 0.1 & 0.2 & 0.02 & 5.88 \\
\hline 112 & B & 4 & $\begin{array}{l}\text { Paramphidelus pusillus (Thorne, 1936) } \\
\text { MONONCHIDA }\end{array}$ & 0.1 & 0.5 & 0.04 & 5.88 \\
\hline 113 & $\mathrm{P}$ & 4 & Clarkus papillatus (Bastian, 1865) & 1.2 & 2.0 & 0.44 & 35.29 \\
\hline 114 & $\mathrm{P}$ & 4 & Coomansus parvus (de Man, 1880) & 2.8 & 4.3 & 1.07 & 52.94 \\
\hline 115 & $\mathrm{P}$ & 4 & Mononchus aquaticus Coetzee, 1968 & 0.1 & 0.5 & 0.04 & 5.88 \\
\hline 116 & $\mathrm{P}$ & 4 & Prionchulus punctatus Cobb, 1917 & 1.2 & 2.1 & 0.44 & 29.41 \\
\hline 117 & $\mathrm{P}$ & 4 & Mylonchulus brachyuris (Bütschli, 1873) & 2.4 & 6.2 & 0.91 & 29.41 \\
\hline 118 & $\mathrm{P}$ & 4 & Mylonchulus sigmaturus (Cobb, 1917) & 1.9 & 2.8 & 0.73 & 41.18 \\
\hline 119 & $\mathrm{P}$ & 4 & $\begin{array}{l}\text { Anatonchus tridentatus (de Man, 1876) } \\
\text { DORYLAIMIDA }\end{array}$ & 3.0 & 4.0 & 1.13 & 64.71 \\
\hline 120 & $\mathrm{O}$ & 4 & Dorylaimus stagnalis Dujardin, 1845 & 1.0 & 4.1 & 0.38 & 5.88 \\
\hline
\end{tabular}




\begin{tabular}{|c|c|c|c|c|c|c|c|}
\hline 121 & $\mathrm{O}$ & 5 & Prodorylaimus acris (Thorne, 1936) & 1.2 & 3.7 & 0.44 & 17.65 \\
\hline 122 & $\mathrm{O}$ & 5 & Mesodorylaimus aduncus Andrássy, 1986 & 0.2 & 1.0 & 0.09 & 5.88 \\
\hline 123 & $\mathrm{O}$ & 5 & Mesodorylaimus bastiani (Bütschli, 1873) & 2.3 & 5.2 & 0.87 & 41.18 \\
\hline 124 & $\mathrm{O}$ & 5 & Mesodorylaimus centrocercus (de Man, 1880) & 0.9 & 3.4 & 0.33 & 11.76 \\
\hline 125 & $\mathrm{O}$ & 5 & Mesodorylaimus meyli Andrássy, 1958 & 0.2 & 0.6 & 0.09 & 17.65 \\
\hline 126 & $\mathrm{O}$ & 5 & Aporcelaimellus obtusicaudatus (Bastian, 1865) & 6.1 & 5.9 & 2.29 & 82.35 \\
\hline 127 & $\mathrm{O}$ & 5 & Paraxonchium laetificans (Andrássy, 1956) & 0.4 & 1.1 & 0.13 & 11.76 \\
\hline 128 & $\mathrm{O}$ & 4 & Eudorylaimus carteri (Bastian, 1865) & 2.2 & 5.6 & 0.82 & 35.29 \\
\hline 129 & $\mathrm{O}$ & 4 & Eudorylaimus confusus/longicardius Thorne, 1974 & 1.0 & 2.5 & 0.38 & 29.41 \\
\hline 130 & $\mathrm{O}$ & 4 & Eudorylaimus leuckarti (Bütschli, 1973) & 0.2 & 1.0 & 0.09 & 5.88 \\
\hline 131 & $\mathrm{O}$ & 4 & Eudorylaimus spp. juvs. & 4.6 & 5.5 & 1.73 & 64.71 \\
\hline 132 & $\mathrm{O}$ & 4 & Microdorylaimus longicollis (Brzeski, 1964) & 0.5 & 2.2 & 0.20 & 5.88 \\
\hline 133 & $\mathrm{O}$ & 4 & Microdorylaimus parvus (de Man, 1880) & 0.1 & 0.5 & 0.04 & 5.88 \\
\hline 134 & $\mathrm{O}$ & 4 & Allodorylaimus granuliferus (Cobb, 1893) & 2.9 & 6.0 & 1.09 & 41.18 \\
\hline 135 & $\mathrm{O}$ & 4 & Thonus ettersbergensis (de Man, 1885) & 1.2 & 1.3 & 0.47 & 58.82 \\
\hline 136 & PP & 4 & Longidorella microdora (de Man, 1880) & 0.5 & 1.9 & 0.18 & 5.88 \\
\hline 137 & $\mathrm{O}$ & 4 & Pungentus engadinensis (Altherr, 1950) & 0.1 & 0.2 & 0.02 & 5.88 \\
\hline 138 & $\mathrm{O}$ & 4 & Pungentus silvestris (de Man, 1912) & 2.9 & 4.8 & 1.11 & 52.94 \\
\hline 139 & $\mathrm{O}$ & 4 & Enchodelus macrodorus (de Man, 1880) & 5.3 & 7.0 & 2.00 & 58.82 \\
\hline 140 & $\mathrm{O}$ & 4 & Enchodelus (Paraenchodelus) sp. & 0.1 & 0.5 & 0.04 & 5.88 \\
\hline 141 & PP & 5 & Longidorus elongatus (de Man, 1876) & 0.1 & 0.5 & 0.04 & 5.88 \\
\hline 142 & $\mathrm{O}$ & 5 & Axonchium cf. deconincki Nair, 1975 & 1.1 & 1.7 & 0.40 & 35.29 \\
\hline 143 & $\mathrm{O}$ & 5 & Axonchium coronatum (de Man, 1906) & 2.0 & 5.4 & 0.75 & 29.41 \\
\hline 144 & $\mathrm{O}$ & 5 & Axonchium nairi Altherr, 1974 & 1.1 & 3.3 & 0.40 & 11.76 \\
\hline 145 & $\mathrm{O}$ & 5 & Oxydirus oxycephalus (de Man, 1885) & 5.8 & 5.1 & 2.17 & 76.47 \\
\hline 146 & $\mathrm{~F}$ & 4 & Tylencholaimus constrictus Vinciguerra, 1986 & 5.9 & 19.1 & 2.24 & 29.41 \\
\hline 147 & $\mathrm{~F}$ & 4 & Tylencholaimus minutus Vinciguerra, 1986 & 0.1 & 0.5 & 0.04 & 5.88 \\
\hline 148 & $\mathrm{~F}$ & 4 & Tylencholaimus mirabilis (Bütschli, 1873) & 0.5 & 1.5 & 0.18 & 11.76 \\
\hline 149 & $\mathrm{~F}$ & 4 & Tylencholaimus sp. juvs. & 0.1 & 0.5 & 0.04 & 5.88 \\
\hline 150 & $\mathrm{~F}$ & 4 & Tylencholaimellus striatus Thorne, 1939 & 0.1 & 0.5 & 0.04 & 5.88 \\
\hline 151 & $\mathrm{O}$ & 4 & Dorylaimoides micoletzkyi (de Man, 1921) & 0.6 & 1.8 & 0.24 & 11.76 \\
\hline 152 & $\mathrm{P}$ & 5 & Nygolaimus trophurus Heyns, 1968 & 0.1 & 0.3 & 0.04 & 11.76 \\
\hline 153 & $\mathrm{P}$ & 5 & Paravulvus hartingii (de Man, 1880) & 0.3 & 0.7 & 0.11 & 17.65 \\
\hline 154 & $\mathrm{P}$ & 5 & $\begin{array}{l}\text { Paravulvus acuticaudatus (Thorne, 1930) } \\
\text { TRIPLONCHIDA }\end{array}$ & 0.1 & 0.2 & 0.02 & 5.88 \\
\hline \multirow[t]{2}{*}{155} & $\mathrm{~F}$ & 3 & Diptherophora communis de Man, 1880 & 2.6 & 4.3 & 1.00 & 52.94 \\
\hline & & & Total nematodes & 265.1 & 172.7 & 100 & 100 \\
\hline
\end{tabular}

\section{Results}

In the meadows studied, 155 species and 86 genera of nematodes in the total material of 4507 individuals were found (Table 1). In the Čertoryje, for the whole period of the investigations there was found 119 species and 79 genera with H'spp $=3.64$ and H'gen $=3.31$. Most nematodes belonged to the orders Tylenchida (52 species and 1824 individuals), Dorylaimida (35 and 888) and Rhabditida (16 and 899). Bacterivores were represented by 47 species and 1180 individuals followed by plant parasites (29 species and 861 individuals), omnivores (25 and 747), root-fungal feeders (22 and 919), fungivores (17 and 526), predators (12 and 264), and insect parasites (3 and 10).

Table 2 shows the values of community indices in the sites studied and Table 3 gives Pearson correlation coefficient between these indices. The number of nematode species at individual sites and sampling dates varied from 18 to 68 , the number of genera from 18 to 52 . Values of diversity in- dices (richness, $\mathrm{H}^{\prime}$, D) for species as well as for genera reached high values, except for the locality 10 with predominance of Helicotylenchus digonicus. The high values were also found for trophic diversity indices $\mathrm{H}$ 'tg and $\mathrm{T}$ (exception the locality 10). The values of MI, PPI and $\Sigma \mathrm{MI}$ were always greater than 2 whereas the ratio PPI/MI equaled or decreased less than 1. Maturity Index had no significant correlation with any diversity index, PPI and $\Sigma \mathrm{MI}$ showed some significant negative correlations with diversity indices. PPI/MI ratio showed no significant correlation with any community index.

CI values were high except for some sites $(5,7,9)$. EI values were significantly negatively correlated with CI values $(\mathrm{r}=-0.72, \mathrm{p}=0.001)$ and these two indices showed no significant correlation with any other community index. SI values were high in all sites and were significantly positively correlated with values of $\mathrm{MI}(\mathrm{r}=0.76, \mathrm{P}<0.001)$ and 


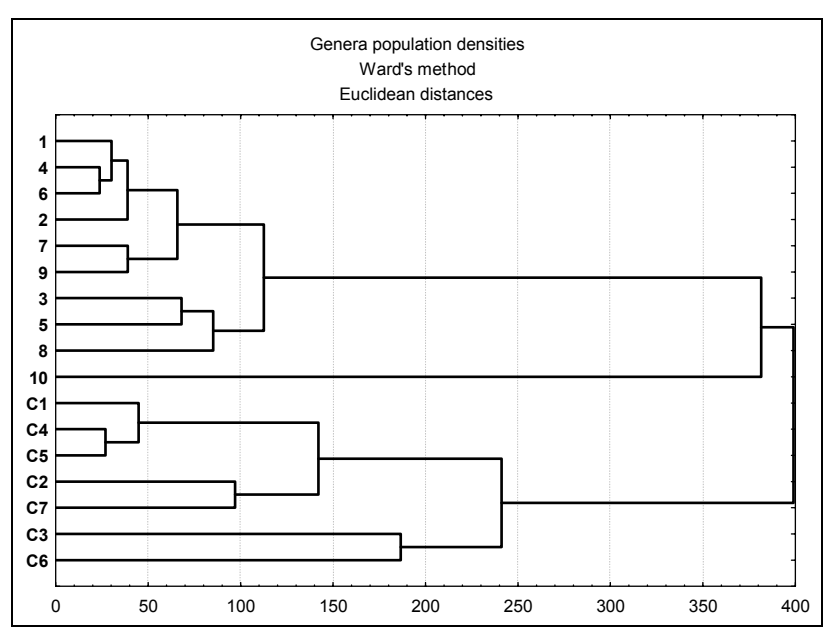

Fig. 1. Cluster analysis of soil nematodes in meadows of the White Carpathians with $\Sigma$ MI $(\mathrm{r}=0.71, \mathrm{p}=0.002)$ but gave no significant correlation with other indices.

Cluster analysis performed on genera population densities (Fig. 1) showed sites on the eastern side of the White Carpathians clearly separated from the group of samples taken in the Čertoryje meadow on the western side. Position of the site 10 was influenced by a high population of Helicotylenchus digonicus.

\section{Discussion}

The number of nematode species in the meadows of the study area was high. For example, Šály (1985) found 96 species in meadows and pastures of the Slovak Paradise and 182 species in its whole territory. Lišková and Čerevková (2005) found 69 species and 48 genera of soil nematodes in five meadow habitats in Slovakia and Zolda (2002)

Table 2. Community indices of soil nematodes in meadows of the White Carpathians, for the Čertoryje meadow mean, \pm S.D. $(n=7)$, and range of values are given

\begin{tabular}{lcccccccccccc}
\hline & 1 & 2 & 3 & 4 & 5 & 6 & 7 & 8 & 9 & 10 & \multicolumn{2}{c}{ Certoryje } \\
(C1 - C7)
\end{tabular}

Table 3. Person correlation coefficients between community indices of nematodes at individual sites and sampling dates, $\mathrm{n}=17$, ${ }^{*} \mathrm{P}<0.05,{ }^{* *} \mathrm{P}<0.01,{ }^{* * *} \mathrm{P}<0.001$

\begin{tabular}{|c|c|c|c|c|c|c|c|c|c|c|c|c|}
\hline Species & SR & H'spp & Dspp & Genera & GR & H'gen & Dgen & $\mathrm{H}^{\prime} \operatorname{tg}$ & $\mathrm{T}$ & $\mathrm{MI}$ & PPI & $\sum \mathrm{MI}$ \\
\hline Individuals $+0.76^{* * *}$ & $+0.61^{* *}$ & +0.12 & -0.05 & $+0.75^{* * *}$ & $+0.55^{*}$ & +0.06 & -0.13 & +0.01 & +0.05 & -0.23 & -0.25 & -0.34 \\
\hline Species & $+0.98^{* * *}$ & $+0.62^{* *}$ & +0.32 & $+0.99^{* * *}$ & $+0.95^{* * *}$ & $+0.54^{*}$ & +0.15 & +0.46 & +0.42 & -0.37 & $-0.59^{*}$ & $-0.56^{*}$ \\
\hline SR & & $+0.72^{* *}$ & +0.40 & $+0.97^{* * *}$ & $+0.99^{* * *}$ & $+0.63^{* *}$ & +0.21 & $+0.56^{*}$ & $+0.50^{*}$ & -0.39 & $-0.66^{* *}$ & $-0.59^{*}$ \\
\hline H'spp & & & $+0.81^{* * *}$ & $+0.62^{* *}$ & $+0.73^{* * *}$ & $+0.98^{* * *}$ & $+0.71^{* *}$ & $+0.91^{* * *}$ & $+0.82^{* * *}$ & -0.46 & $-0.59^{*}$ & -0.47 \\
\hline Dspp & & & & +0.30 & +0.40 & $+0.83^{* * *}$ & $+0.95^{* * *}$ & $+0.78^{* * *}$ & $+0.77^{* * *}$ & -0.26 & -0.16 & -0.14 \\
\hline Genera & & & & & $+0.96^{* * *}$ & $+0.54^{*}$ & +0.14 & +0.46 & +0.41 & -0.32 & $-0.57^{*}$ & $-0.52^{*}$ \\
\hline GR & & & & & & $+0.65^{* *}$ & +0.23 & $+0.58^{*}$ & $+0.52^{*}$ & -0.32 & $-0.63^{* *}$ & $-0.52^{*}$ \\
\hline H'gen & & & & & & & $+0.79^{* * *}$ & $+0.91^{* * *}$ & $+0.83^{* * *}$ & -0.41 & $-0.48^{*}$ & -0.36 \\
\hline Dgen & & & & & & & & $+0.72^{* *}$ & $+0.73^{* * *}$ & -0.16 & +0.03 & +0.03 \\
\hline H'tg & & & & & & & & & $+0.97^{* * *}$ & -0.29 & $-0.48^{*}$ & -0.32 \\
\hline $\mathrm{T}$ & & & & & & & & & & -0.18 & -0.38 & -0.21 \\
\hline MI & & & & & & & & & & & $+0.67^{*}$ & $+0.90^{* * *}$ \\
\hline PPI & & & & & & & & & & & & $+0.86^{* * *}$ \\
\hline
\end{tabular}


determined 21 genera in the rhizosphere of meadows in the National Park See Neusiedl-Seewinkel in Austria. In Spain, Dmowska (2000) identified 140 nematode species from 71 genera in the rhizosphere of subalpine meadows and evaluated the dominance of nematode taxa according to different soil types and climates of the investigated regions of Pyrenees. Hodda and Wanless (1994) recorded 154 nematode species in English chalk grasslands. Wasilewska (2002) identified 122 genera of soil nematodes on fen peat meadows in Biebrza Wetlands in Poland over a long period of investigation since 1978 to 1997 . As seen, the number of species and genera vary with site and time. In the Čertoryje meadow the mean number of species on individual sampling dates represented $45 \%$ of all species found in the locality. In four different ecosystems studied by Háněl (1995) the mean number of species over sampling dates in individual years varied from $45 \%$ to $63 \%$ of the year total. We can infer that one-date sampling can reveal about a half of the total soil nematode species in a locality; in genera the proportion can be greater.

This is in agreement with Table 3 where the number of individuals is significantly positively correlated with the number of species and genera as well as SR and GR. On the other hand, other diversity indices showed low insignificant correlations with number of individuals. H'spp was significantly positively correlated with all diversity indices as well as H'gen and both can be used for evaluation of heterogeneity of a nematode assemblage. H'tg and T changed in similar way being little affected by the number of individuals, species and genera. Therefore, the number of species \& H'spp (genera \& H'gen) together with T can be sufficient for characterization of taxonomic and trophic diversity of a nematode assemblage; taking into consideration that the number of species (genera) can be influenced by the number of individuals studied. For this reason the number of nematode individuals determined should be also provided.

MI was negatively but insignificantly correlated with diversity indices, but the respective correlations for PPI were mostly negative significant, which affected results obtained for $\Sigma$ MI (which includes PPI taxa). Explanation of this phenomenon is problematic. In succession studied by Háněl (1995) MI was positively correlated with the number of species, H'spp and H'gen whereas PPI was correlated negatively with these diversity indices. The diversity of soil nematodes in succession increased but simultaneously with increase in populations of Tylenchidae with c-p 2, which decreased the values of PPI. At least some Filenchus species (such as $F$. misellus and $F$. discrepans) are fungivorous and their high population densities can affect the results of nematode community analyses (Okada et al. 2005).

$\mathrm{PPI} / \mathrm{MI}$ ratio was lower or equal to 1 , which according to Bongers et al. (1997) indicates natural habitats, where plants make optimal use of nutrient resources or only slight nutrient disturbances. Generally high values of CI also indicated dominance of fungal pathways in decomposition food webs in the meadows studied and their undisturbed conditions (Bardgett \& McAlister, 1999). Low CI in the sites 5,7 , and 9 were caused by temporarily high population densities of Rhabditis, which occur in patches of the intensive organic matter decay. The values of EI and CI determined position of the sites studied in the quadrants $\mathrm{B}$ and $\mathrm{C}$ as defined by Ferris et al. (2001). The position of sites in these quadrants is and indication of maturing structuredstable soil food-web conditions with low degree of disturbance. Also diversity indices of nematodes varied in similar range on both sides of the White Carpathians.

The high values of MI, SI as well as diversity indicated structured nematode assemblages. However, these assemblages were taxonomically different as shown by cluster analysis (Fig. 1). This implies that a structured-stable food web in soil can be maintained by different species composition of nematodes. Ritz and Trudgill (1999) suggested that studies of nematodes should focus on functional level (trophic groups, $\mathrm{r} / \mathrm{K}$ strategists ratio, rates of population increase, etc.) rather than at the family, generic or species level, although they mentioned 'key' species/genera and alltaxa biotic inventory. Yeates (2003) stated that allocation of nematodes to trophic and c-p groups is often uncertain and proposed species-level discrimination as necessary to permit further advances in understanding the role of nematodes in the soil. Results of our study suggest that dominant species (genera) in each functional group should be determined. For example, species of the families Tylenchidae and Tylenchulidae are both included in PPI group and c-p value 2 . But the family Tylenchidae represents facultative/obligatory plant feeders, which does not cause serious damage to plants whereas species of the family Tylenchulidae can have very negative influence on plant growth. Therefore, interpretation of the same value of PPI can be different and species dependent.

\section{Acknowledgements}

The study was carried in the frame of the IRP ISB AS CR AV0Z60660521, the joint project "Soil nematodes (Nematoda) of meadow ecosystems of the Czech Republic and the Slovak Republic" between ISB AS CR and PI SAS and by scientific grant agency VEGA, No. 2/4176/04.

\section{References}

BArdgett, R. D., McAlister, E. (1999): The measurement of soil fungal: bacterial biomass ratios as an indicator of ecosystem self-regulation in temperate meadow grasslands. Biol. Fertil. Soils, 29: $282-290$

BONGERS, T. (1990): The maturity index: an ecological measure of environmental disturbance based on nematode species composition. Oecologia, 83: $14-19$

Bongers, T., Van Der Meulen, H., Korthals, G. (1997): Inverse relationship between the nematode maturi-ty index and plant parasite index under enriched nutrient conditions. Appl. Soil Ecol., 6: 195 - 199

DMOWSKA, E. (2000): Nematode communities in subalpine meadows in Central Pyrenees, Ann. Zool., 50: 211 - 220

EKSChMitT, K., BAKOnYI, G., Bongers, M., Bongers, T., Boström, S., Dogan, H., Harrison, A., Nagy, P., 
O’donnell, A. G., Papatheodorou, E. M., Sohlenius, B., Stamou, G. P., Wolters, V. (2001): Nematode community structure as indicator of soil functioning in European grassland soils. Eur. J. Soil Biol., 37: $263-268$

Ferris, H., Bongers, T., DE Goede, R. G. M. (2001): A framework for soil food web diagnostics: extension of the nematode faunal analysis concept. Appl. Soil Ecol., 18: $13-$ 29

Freckman, D. W., EtTema, C. H. (1993): Assessing nematode communities in agroecosystems of varying human intervention. Agric. Ecosyst. Environ., 45: 239 - 261

HÁNĚL, L. (1995): Secondary successional stages of soil nematodes in cambisols of South Bohemia. Nematologica, 41: $197-218$

HÁNĚL, L. (2003): Recovery of soil nematode populations from cropping stress by natural secondary succession to meadow land. Appl. Soil Ecol., 22: $255-270$

HodDA, M., WANLESS, F. R. (1994): Nematodes from an English chalk grassland: Species distributions. Nematologica, 40: $116-132$

LiŠKovÁ, M., ČEREVKOVÁ, A. (2005): Nematode communities of river banks and adjacent meadows in the Slovak Republic. Helminthologia, 42: 223 - 232

OKADA, H., HARADA, H., KADOTA, I. (2005): Fungal-feeding habits of six nematode isolates in the genus Filenchus. Soil Biol. Biochem., 37: 1113 - 1120

RECEIVED NOVEMBER 16, 2005
Ritz, K., TrudgiLl, D. L. (1999): Utility of nematode community analysis as an integrated measure of the functional state of soils: perspectives and challenges. Plant Soil, 212: $1-11$

ŠÁLY, A. (1985): Production of free living nematodes in the Protected landscape area of the Slovak Paradise. Eko-lógia (ČSSR), 4: 185 - 209

StATsoft, InC. (2001): STATISTICA Cz [Softwarový systém na analýzu dat], verze 6 . [Software system for data analysis], Release 6], Www.StatSoft.Cz, (in Czech and English)

WASILEWSKA, L. (2002): Post-drainage secondary succession of soil nematodes on fen peat meadows. Pol. J. Ecol., 50: $269-300$

YEATES, G. W. (2003): Nematodes as soil indicators: functional and biodiversity aspects. Biol. Fertil. Soils, 37: $199-$ 210

YeAtes, G. W., BONGERS, T. (1999): Nematode diversity in agroecosystems. Agric. Ecosyst. Environ., 113 - 135

Yeates, G. W., Bongers, T., De Goede, R. G. M., FreckMAN, D. W., GeOrgIEVA, S. S. (1993): Feeding habits in nematode families and genera - an outline for soil ecologists. J. Nematol., 25: $315-331$

ZOLDA, P. (2002): Untersuchungen zur Nematodenfauna der Trockengrasen im Nationalpark Neusiedler See - Seewinkel. Verh. Zool.-Bot. Ges. Österreich, 139: 53 - 58 